\title{
Efecto del residual de estiércol avícola o residual de fertilizante mineral en el rendimiento y la calidad de camelina (Camelina sativa L. Crantz)
}

\section{Effect of residual poultry manure or residual mineral fertilizer on yield and quality of camelina (Camelina sativa L. Crantz)}

\author{
Rosario Miralles de Imperial Hornedoa, María Mar Delgado Arroyo , Ángela García Mansoa, \\ María I sabel González Gullóna , J osé Valero Martín Sánchez*
}

\begin{abstract}
RESUMEN
El interés por el cultivo de camelina [Camelina sativa (L.) Crantz] para alimentación animal y biocombustible se está incrementando. Se condujo un ensayo en invernadero y tiestos con camelina cv. Calena después de un primer cultivo de cebada ( Hordeum vulgare L.), con el fin de estudiar el efecto de la fertilización residual de gallinazas y mineral en el rendimiento y la calidad del grano. Los tratamientos aplicados en el cultivo anterior de cebada fueron: gallinaza o fertilizante mineral, N-P-K en dosis $\mathbf{0}$, $\mathbf{1}$, 1.5 y 2 (0, 60, 90,120 unidades fertilizantes de nitrógeno). Las variables estudiadas fueron: peso seco de biomasa aérea, peso seco de grano, peso de 1,000 granos, porcentaje de nitrógeno en biomasa aérea, porcentaje de nitrógeno en grano, porcentaje de proteína bruta en grano y porcentaje de grasa bruta en grano. Los tratamientos y dosis fueron significativamente diferentes. La interacción tratamiento* dosis fue significativa $(P<0.05)$ para las variables estudiadas excepto para peso grano y peso de 1,000 granos. Para la gallinaza y el mineral los valores más altos en peso de grano se obtuvieron con dosis de 1 y 1.5 , con rendimientos equivalentes en grano de 1,547 kg/ ha y 1,504 kg/ ha, para proteína bruta en grano fueron para dosis 1.5 en ambos tratamientos con 455.08 y 478.72 kg/ ha y para grasa bruta del grano fueron los más altos, para dosis de 1 y 1.5 con 481.27 y 454.06 kg/ ha. En general, la camelina tuvo una buena respuesta a la fertilización residual con fertilización a partir de la gallinaza o mineral en rendimiento y calidad de grano.
\end{abstract}

PALABRAS CLAVE: Aceite, Cultivo, Fertilización, Gallinaza, Proteína.

\begin{abstract}
The growing interest in camelina (Camelina sativa L. Crantz) is increasing for animal nutrition and for biofuel. A trial was carried out in a greenhouse with camelina cv. 'Calena' cultivated in pots in order to evaluate the effect of residual poultry manure and mineral fertilization after a crop of barley (Hordeum vulgare L.) and to determine the grain's yield and quality. The treatments applied to the first culture of barley were two: poultry manure or mineral fertilizer N-P-K, at rates of $0,1,1.5$ and $2(0,60,90,120$ nitrogen fertilizing units). The variables analyzed were the following: dry weight of: seed and aerial biomass and 1,000 seeds; percentages of Kjeldhal nitrogen in: seed, and aerial biomass; percentages of: crude protein and oil content. Treatment type and rates were significant. Treatment* rate interaction was significant $(P<0.05)$ for the analyzed variables except for weight of seed and weight of 1,000 seeds. For the laying hen or mineral the highest values in terms of grain weight were obtained with rates 1 and 1.5, with grain yields equivalent of 1,547 and 1,504 kg/ ha, respectively. I $\mathrm{n}$ terms of crude protein grain the highest values were obtained with rate 1.5 for both treatments, with protein yield equivalent of 455.08 and $478.72 \mathrm{~kg} / \mathrm{ha}$ and in terms of oil content seed the highest values were obtained with rates 1 and 1.5 with oil content yield of 481.27 and $454.06 \mathrm{~kg} / \mathrm{ha}$. In general, camelina had a good response to residual poultry manure or mineral fertilizer in yield and quality of grain.
\end{abstract}

KEY WORDS: Crop, Poultry manure, Fertilization, Oil, Protein.

Recibido el 4 de junio de 2016. Aceptado el 20 de diciembre de 2016.

a Departamento de Medio Ambiente. Instituto Nacional de Investigación y Tecnología Agraria y Alimentaria (INIA). España.

*Autor de correspondencia: vmartin@inia.es 


\section{INTRODUCCIÓN}

La camelina (Camelina sativa L. Crant) de la familia Brassicaceae, aunque es un antiguo cultivo que se conocía desde la época de los romanos ${ }^{(1)}$ es una planta cuyo cultivo se está incrementando estos últimos años en España ${ }^{(2)}$ y otros países europeos como Rumania(3,4), Dinamarca( ${ }^{(5)}$, Noruega ${ }^{(6)}$ y en países de América del norte y del sur como Estados Unidos $^{(7)}$, Canadá( ${ }^{(8,9,10)}$ y Chile ${ }^{(1,11)}$.

Esta extensa implantación por todo el mundo es debida a sus buenas características y variadas potencialidades, ya que posee una facilidad de cultivo y recolección sin necesidad de maquinaria especializada, y por sus posibilidades tanto en alimentación animal|(12) y alimentación humana ${ }^{(5)}$, por su alto contenido en ácidos grasos poliinsaturados omega 3 , omega 6 y aminoácidos ${ }^{(13)}$; también por su bajo contenido en glucosilonatos ${ }^{(6)}$, así como también para el uso de su aceite como biocombustible para aviones ${ }^{(4)}$, por el alto contenido de aceite en sus semillas.

Por todo ello, en los últimos años existe un renovado interés de este cultivo y se ha venido estudiando su respuesta a la aplicación de fertilizantes nitrogenados en distintas dosis y el efecto en su rendimiento, contenido en nitrógeno y calidad del grano ${ }^{(8,14)}$.

La aplicación de estiércoles de granjas avícolas como fertilizante orgánico para los cultivos es una práctica idónea para una agricultura sostenible, se aprovecha su contenido en nutrientes disponibles y el aporte de su materia orgánica supone una mejora para el suelo cultivado, pero siempre que se controlen las dosis aplicadas de estos estiércoles para obtener mejoras en los rendimientos y calidad de los cultivos, y a su vez sean ambientalmente correctas, evitando la contaminación de los mantos acuíferos y los suelos. La aplicación de la gallinaza como fertilizante a los cultivos ha sido estudiada estos últimos años en ensayos de invernadero y macetas en maíz ${ }^{(15)}$ y sorgo forrajero(16)

Además la utilización de la gallinaza para fertilizar, permite el cierre de ciclo, al usar el grano de la camelina en la alimentación de las gallinas, y así obtener huevos de composición diferente en omega 3 y omega 6 , alcanzándose un producto diferenciado dentro del mercado.

La cantidad y características de la gallinaza dependen de la edad, la dieta y la salud de las aves, así como de las prácticas de gestión agrícola. Las estimaciones de excretas por 1,000 aves al día (basadas en el promedio de peso diario vivo durante el ciclo de producción de las aves), se sitúan en torno a $120 \mathrm{~kg}$ para las gallinas ponedoras, y $80 \mathrm{~kg}$ para los pollos de carne ${ }^{(17)}$. La cantidad y composición de la gallinaza depende de las diferentes prácticas de producción avícola y es fundamental para una gestión eficiente y ambientalmente responsable de estos subproductos para la fertilización del suelo.

Una característica importante del cultivo de la camelina es que puede fácilmente formar parte en las rotaciones de secano y regadío, y aprovechar la fertilización residual del cultivo anterior. La información del efecto residual de la aplicación de la gallinaza en una rotación de cultivos y su aprovechamiento en un cultivo posterior es limitada.

Con el objetivo de evaluar el efecto residual de la aplicación de la excreta de la gallina de postura y compararlo con una fertilización mineral, aplicados ambos tratamientos fertilizantes a un cultivo anterior de cebada, se planteó un ensayo en invernadero y tiestos o recipientes con camelina cv. 'Calena' en siembra otoñal para estudiar el efecto de estos residuales en su rendimiento y en varios parámetros de calidad.

\section{MATERIAL Y MÉTODOS}

Los tiestos con suelos y el residual de gallinaza o residual de fertilizante mineral que se reutilizaron en el presente ensayo, provenían de un primer ensayo que se realizó en cultivo de cebada (Hordeum vulgare L.) en un invernadero localizado en Madrid capital. Los contenedores eran de polipropileno flexible de $26 \mathrm{~L}$ de capacidad y $0.3 \mathrm{~m}$ de profundidad. Se rellenaron con $26 \mathrm{~kg}$ de suelo procedente de la finca del INIA de "La Canaleja" (Madrid), que previamente se habían mezclado con las dosis de gallinaza de gallina ponedora estabulada o de fertilizante mineral. El tiesto lleno de suelo y 
gallinaza o suelo y fertilizante mineral alcanzó una altura media de $0.27 \mathrm{~m}$. La superficie resultante de tiesto una vez lleno fue de $0.1 \mathrm{~m}^{2}$ (diámetro $0.38 \mathrm{~m}$ ). Las características del suelo utilizado se presentan en la Cuadro 1. Los tratamientos fertilizantes aplicados al cultivo de cebada fueron: excreta de gallina ponedora (GP) y fertilizante mineral (FM). Las gallinazas utilizadas en el ensayo provenían de la granja avícola de la finca "El Encín" (Madrid), cuyas características se presentan en el Cuadro 2; su contenido de metales pesados no sobrepasa los límites máximos establecidos por la legislación sobre productos fertilizantes ${ }^{(18)}$.

Para el cálculo del abonado del cultivo de cebada se tuvieron en cuenta sus necesidades de nutrientes, las características del suelo y zona de procedencia de dicho suelo, y para una producción estimada de grano de 2,000 kg/ha se necesitarían aportar $60 \mathrm{~kg}$ nitrógeno. Para la superficie del tiesto de $0.1 \mathrm{~m}^{2}$ en que se iban a desarrollar seis plantas de cebada se necesitaría aplicar $0.6 \mathrm{~g}$ de $\mathrm{N}$ por
Cuadro 1. Características del suelo (materia seca)

\begin{tabular}{lc}
\hline Parámetro & Suelo \\
\hline $\mathrm{pH} 1: 2,5 \mathrm{H}_{2} \mathrm{O}$ & 7.44 \\
Conductividad eléctrica 1:5 $\mathrm{H}_{2} \mathrm{O}$ dSm${ }^{-1}$ & 0.22 \\
Carbono orgánico oxidable, \% & 0.84 \\
Nitrógeno Kjeldahl, \% & 0.09 \\
Relación C/N & 9.33 \\
Materia orgánica oxidable, \% & 1.45 \\
$\mathrm{~N}^{-\mathrm{NH}_{4}}{ }^{+}, \mathrm{mg} / \mathrm{kg}$ & 2.71 \\
$\mathrm{~N}^{-\mathrm{NO}_{3}}, \mathrm{mg} / \mathrm{kg}$ & 2.27 \\
Fósforo (Olsen), mg/kg & 21 \\
Potasio (acetato amónico), mg/kg & 392 \\
\hline
\end{tabular}

tiesto, ésta sería la dosis 1 (d1), que sería la que cubriría las necesidades de N. Las dosis 1.5 (d 1.5) y dosis $2(\mathrm{~d} 2)$ eran 1.5 veces y 2 veces las aplicadas con d1, luego habría que aportar 0.9 y 1.2 g de $\mathrm{N}$ por tiesto respectivamente. Para cubrir las necesidades de $\mathrm{N}$ por tiesto con la dosis 1 de gallinaza (GP-d1) se aplicaron $24.5 \mathrm{~g}$, con la dosis 1.5 de gallinaza (GP-d 1.5) fueron $36.75 \mathrm{~g}$ y con la

Cuadro 2. Características de la gallinaza de gallina ponedora (materia seca)

\begin{tabular}{|c|c|c|c|c|c|c|}
\hline \multicolumn{4}{|l|}{ Parámetro } & \multicolumn{2}{|c|}{ Gallinaza } & \\
\hline \multicolumn{4}{|l|}{ pH } & \multicolumn{3}{|c|}{7.9} \\
\hline \multicolumn{4}{|l|}{$\mathrm{CE}^{\star}, \mathrm{dSm}^{-1}$} & \multicolumn{3}{|c|}{10.2} \\
\hline \multicolumn{4}{|l|}{ Humedad, \% } & \multicolumn{3}{|c|}{65.47} \\
\hline \multicolumn{4}{|c|}{ Sólidos totales, \% } & \multicolumn{3}{|c|}{36.88} \\
\hline \multicolumn{4}{|c|}{ Sólidos fijos, \% } & \multicolumn{3}{|c|}{11.81} \\
\hline \multicolumn{4}{|c|}{ Sólidos volátiles, \% } & \multicolumn{3}{|c|}{25.07} \\
\hline \multicolumn{4}{|l|}{ N Kjeldahl, \% } & \multicolumn{3}{|c|}{3.97} \\
\hline \multicolumn{4}{|l|}{$\mathrm{N}-\mathrm{NH}_{4}+, \mathrm{mg} / \mathrm{kg}$} & \multicolumn{3}{|c|}{22,862} \\
\hline \multicolumn{4}{|l|}{$\mathrm{N}-\mathrm{NO}_{3}, \mathrm{mg} / \mathrm{kg}$} & \multicolumn{3}{|c|}{265} \\
\hline \multicolumn{4}{|c|}{ C orgánico oxidable, $\%$} & \multicolumn{3}{|c|}{30.38} \\
\hline \multicolumn{4}{|c|}{$\mathrm{P}, \mathrm{mg} / \mathrm{kg}$} & \multicolumn{3}{|c|}{13,556} \\
\hline \multicolumn{4}{|l|}{$\mathrm{K}, \mathrm{mg} / \mathrm{kg}$} & \multicolumn{3}{|c|}{26,632} \\
\hline \multicolumn{4}{|l|}{$\mathrm{Ca}, \mathrm{mg} / \mathrm{kg}$} & \multicolumn{3}{|c|}{114,555} \\
\hline \multicolumn{4}{|l|}{$\mathrm{Mg}, \mathrm{mg} / \mathrm{kg}$} & \multicolumn{3}{|c|}{7,091} \\
\hline \multicolumn{4}{|l|}{$\mathrm{Na}, \mathrm{mg} / \mathrm{kg}$} & \multicolumn{2}{|c|}{4,658} & \\
\hline \multicolumn{7}{|c|}{$\begin{array}{l}\text { Concentración de metales pesados }\left(\mathrm{mg} \mathrm{kg}^{-1} \text { de la gallinaza y límites máximos de metales pesados }{ }^{\star \star} \text { para productos }\right. \\
\text { fertilizantes elaborados con residuos orgánicos }\end{array}$} \\
\hline Metal pesado & $\mathrm{Cd}$ & $\mathrm{Cr}$ & $\mathrm{Cu}$ & $\mathrm{Pb}$ & $\mathrm{Ni}$ & $\mathrm{Zn}$ \\
\hline Gallinaza & 0 & 9 & 41.1 & 0 & 4.8 & 189.1 \\
\hline${ }^{\star *}$ Clase A & 0.7 & 70 & 70.0 & 45 & 25.0 & 200.0 \\
\hline${ }^{\star *}$ Clase B & 2.0 & 250 & 300.0 & 150 & 90.0 & 500.0 \\
\hline${ }^{\star \star}$ Clase C & 3.0 & 300 & 400.0 & 200 & 100.0 & 1000.0 \\
\hline
\end{tabular}

${ }^{*} \mathrm{CE}=$ conductividad eléctrica.

**BOE. 2013. Real Decreto 506/2013 de 28 de junio sobre productos fertilizantes. 
dosis 2 de gallinaza (GP-d2) se añadieron $49 \mathrm{~g}$. El fertilizante mineral (FM) utilizado en el ensayo fue un N-P-K, 15-15-15, y se aplicó por tiesto: $4 \mathrm{~g}$ para la dosis 1 (FM-d1), $6 \mathrm{~g}$ para la dosis 1.5 (FM-d1.5) y $8 \mathrm{~g}$ para la dosis 2 (FM- d2). Para las dosis 0 (d0), testigo sin fertilización, fueron $0 \mathrm{~g}$ tanto con GP como FM. Las equivalencias de estas dosis a nivel agronómico se pueden ver en el Cuadro 3.

En el primer cultivo de cebada la siembra se efectuó a mediados de noviembre de 2014 y la recolección se realizó en los primeros días de julio de 2014. Después de la recolección del cultivo de la cebada y tras un periodo de descanso (tres meses), de estos suelos sin añadir fertilizantes de nuevo, se procedió a la toma de muestras de los mismos para su análisis previo a la siembra del cultivo de camelina, para así conocer las características físicoquímicas de estos suelos iniciales con el residual de la excreta de gallina ponedora o el residual de fertilizante mineral y sus respectivas dosis ( $d 0, d 1, d$ 1.5 y d2) para el desarrollo de la camelina. Las características de estos suelos se presentan en el Cuadro 4.

A mediados de octubre de 2014 en los mismos tiestos y suelos con el residual de gallinaza o de fertilizante mineral y sus respectivas dosis en que se había realizado el cultivo de cebada, se procedió a sembrar la camelina como segundo cultivo. La

Cuadro 3. Fertilización aplicada en los cultivos

\begin{tabular}{cccc}
\hline Cultivo & Dosis & \multicolumn{2}{c}{ Fertilizante kg/ha } \\
\hline \multirow{4}{*}{ Cebada } & & Gallinaza & Fertilizante mineral \\
& $\mathrm{d} 0$ & 0 & 0 \\
& $\mathrm{~d} 1$ & 2,450 & 400 \\
& $\mathrm{~d} 1.5$ & 2,675 & 600 \\
& $\mathrm{~d} 2$ & 4,900 & 800 \\
& $\mathrm{~d} 0$ & 0 & 0 \\
Camelina & $\mathrm{d} 1$ & 0 & 0 \\
& $\mathrm{~d} 1.5$ & 0 & 0 \\
& $\mathrm{~d} 2$ & 0 & 0 \\
\hline
\end{tabular}

Cuadro 4. Características de los suelos iniciales

\begin{tabular}{|c|c|c|c|c|c|c|c|}
\hline & STd0 & SGPd1 & SGPd1.5 & SGPd2 & SFMd1 & SFMd1.5 & SFMd2 \\
\hline $\mathrm{pH}, 1: 2.5 \mathrm{H}_{2} \mathrm{O}$ & 7.58 & 7.48 & 7.62 & 7.63 & 7.58 & 7.52 & 7.43 \\
\hline $\begin{array}{l}\mathrm{CE}, 1: 5 \mathrm{H}_{2} \mathrm{O} \\
\mathrm{dSm}{ }^{-1}\end{array}$ & 0.1040 & 0.1031 & 0.1165 & 0.1041 & 0.1173 & 0.1250 & 0.1046 \\
\hline $\begin{array}{l}\text { C orgánico } \\
\text { oxidable, \% }\end{array}$ & 0.8918 & 0.8323 & 0.8624 & 0.7914 & 0.8706 & 0.8865 & 0.8555 \\
\hline $\begin{array}{l}\text { Mat. org. } \\
\text { oxid, \% }\end{array}$ & 1.5339 & 1.4287 & 1.4834 & 1.3612 & 1.4974 & 1.5248 & 1.4714 \\
\hline $\begin{array}{l}\text { Nitrógeno } \\
\text { Kjeldahl, \% }\end{array}$ & 0.005 & 0.1083 & 0.109 & 0.109 & 0.103 & 0.109 & 0.113 \\
\hline Relación C/N & 8.49 & 7.69 & 8.37 & 7.26 & 8.45 & 8.13 & 7.57 \\
\hline $\mathrm{N}-\mathrm{NH}_{4^{+}}, \mathrm{mg} / \mathrm{kg}$ & 3.95 & 4.33 & 6.88 & 3.70 & 3.2 & 1.4 & 0.6 \\
\hline $\mathrm{N}-\mathrm{NO}_{3}^{-}, \mathrm{mg} / \mathrm{kg}$ & 11.59 & 13.53 & 18.93 & 18.03 & 22.3 & 14.12 & 13.87 \\
\hline $\begin{array}{l}\mathrm{P} \text { (Olsen), } \\
\mathrm{mg} / \mathrm{kg}\end{array}$ & 18 & 52 & 52 & 50 & 51 & 55 & 50 \\
\hline $\begin{array}{l}\text { K (acetato } \\
\text { amónico), } \\
\mathrm{mg} / \mathrm{kg}\end{array}$ & 252 & 248 & 255 & 239 & 261 & 263 & 266 \\
\hline
\end{tabular}

Suelo testigo gallinaza dosis 0 (STd0), suelo + gallinaza dosis1 (SGPd1), suelo + gallinaza dosis1.5 (SGPd1.5), suelo + gallinaza dosis 2 (SGPd2), suelo + fertilizante mineral dosis1 (SFMd1), suelo + fertilizante mineral dosis1.5 (SFMd1.5), suelo + fertilizante dosis 2 (SFMd2).

$\mathrm{CE}=$ conductividad eléctrica. 
siembra fue superficial, a $1 \mathrm{~cm}$ de profundidad debido al pequeño tamaño de la semilla. Se sembraron seis semillas por tiesto (de $0.1 \mathrm{~m}^{2}$ de superficie), que corresponderían a una densidad de siembra equivalente de 600,000 plantas/ha. Las características de las semillas de siembra fueron: peso de 1,000 semillas $1.2 \mathrm{~g}$, contenido en aceite expresado en porcentaje de grasa bruta $37.5 \%$ y proteína bruta $25.19 \%$.

El diseño experimental fue totalmente al azar, con arreglo factorial de doble entrada (tratamiento fertilizante, dosis) con tres repeticiones por tratamiento: residual de excreta de gallina ponedora o residual de fertilizante mineral y dosis $(0,1,1.5$ y 2). El número total de tiestos fue de 24.

Durante el tiempo de duración del ensayo se regaron los tiestos según las necesidades hídricas del cultivo, en función de la climatología y por debajo de la capacidad de retención de humedad (CRH) que fue de $4,000 \mathrm{ml}$ (100\% CRH). Mientras duró el ensayo se mantuvo cada tiesto al $60 \%$ de su CRH.

La recolección de la camelina se efectuó cuando alcanzó su madurez fisiológica y el 80 a $90 \%$ de sus silicuas tenían un color entre amarillo y marrón ${ }^{(19)}$, y fue a finales de abril de 2015. Se cortaron las seis plantas de camelina que se habían desarrollado por tiesto. Se separaron la parte aérea del sistema radical. El cuello de la planta fue el punto de corte. Para obtener el peso seco (rendimiento biológico) de estos materiales, se procedió a su secado en estufa a $70 \stackrel{\circ}{ } \mathrm{C}$ hasta peso constante. Se evaluaron sobre este material vegetal, el peso de la biomasa aérea (incluyó tallos, hojas, y las cubiertas de las silicuas), y el peso del grano. También se controló el peso de 1,000 granos, ya que en la familia Brassicaceae es un carácter genético.

Los parámetros evaluados en planta de camelina fueron: producción de grano, peso de biomasa aérea (PBA) y peso de 1,000 granos (P1000G); contenido en nitrógeno en biomasa aérea (\%NBA) y en grano (\%NG), y para valorar la calidad del grano se controló su contenido en proteína bruta y en grasa bruta (contenido en aceite), para así evaluar el posible doble uso de esta semilla en alimentación animal y como materia prima para la obtención de biocarburantes.

En el material vegetal: biomasa aérea y grano, se determinó su contenido en nitrógeno por el método Kjeldahl(20). La proteína bruta en el grano (\%PBG) fue el resultado de multiplicar el porcentaje de nitrógeno en grano (\%NG) por un factor fijo de 6.25 (N Kjeldahl * 6.25). En el grano, sobre materia vegetal seca, se determinó el contenido en aceite como porcentaje de grasa bruta (\%GBG), por el método Soxhlet ${ }^{(21)}$.

Las siete variables estudiadas fueron: peso seco de biomasa aérea $\mathrm{g}$ tiesto ${ }^{-1}$, peso seco de grano $\mathrm{g}$ tiesto-1 $^{-1}$, peso de 1,000 granos, porcentajes de nitrógeno en biomasa aérea, de nitrógeno en grano, de proteína bruta en grano y de grasa bruta en grano.

Para el estudio estadístico de las variables mencionadas se realizó una comprobación de la normalidad de los datos, y posteriormente se procedió a realizar un análisis de varianza (ANOVA) factorial de doble entrada [tratamiento (residual de gallinaza de gallina ponedora (GP) y residual de fertilizante mineral (FM) y dosis (d0, d1 y d1.5) ], y un estudio de interacciones para evaluar la influencia tratamiento*dosis para las variables evaluadas. Los datos se analizaron con el programa Statgraphics Centurión. Para la separación de medias se utilizó el test de rango múltiple de Duncan. Cuando el ANOVA mostró que la interacción fue significativa, se estudió la comparación de medias para las diferentes dosis dentro de cada tratamiento.

\section{RESULTADOS Y DISCUSIÓN}

Los resultados obtenidos en la mayoría de los parámetros estudiados mostraron diferencias significativas entre los tratamientos, siendo un caso de fertilización residual tras un cultivo previo, lo que hace más interesante estas diferencias. Respecto a la producción de camelina se encontraron aumentos frente al testigo, que fueron desde 100 a $300 \mathrm{~kg} / \mathrm{ha}$ y variaciones en proteína bruta y grasa bruta del grano que fueron entre 0.3 a 2.2 y 0.3 a $2.47 \%$ respectivamente, datos que se comentan más detalladamente a continuación y que tienen unos 
efectos importantes en los costes/beneficios y manejo del cultivo.

Se realizó el análisis de varianza para todas las variables estudiadas para comprobar las posibles interacciones (Cuadro 5).
La interacción de tratamiento*dosis, fue significativa para PBA y no significativa para P1000 y PG (Figura 1). Para las variables \%NBA, \%NG, \%PBG y \%GBG, la interacción fue significativa para todas (Figura 2).

Cuadro 5. Análisis de varianza de las variables estudiadas

\begin{tabular}{lccc}
\hline Variable & Tratamiento & Dosis & Tratamiento*dosis \\
\hline Peso biomasa aérea & $*$ & $*$ & $*$ \\
Peso grano & NS & $*$ & NS \\
Peso de 1,000 granos & NS & $*$ & NS \\
Nitrógeno biomasa aérea, $\%$ & NS & $*$ & $*$ \\
Nitrógeno en grano, $\%$ & $*$ & $*$ & $*$ \\
Proteína bruta en grano, \% & $*$ & $*$ & $*$ \\
Grasa bruta en grano, $\%$ & $*$ & $*$ & $*$ \\
\hline
\end{tabular}

NS= no significativo, ${ }^{*}=$ significativo $(P<0.05)$.

Figura 1. Interacción tratamiento (gallinaza o fertilizante mineral) * dosis $(0,1,1,5$ y 2), para peso de grano (rendimiento por tiesto), peso de 1,000 granos y peso de biomasa aérea, de camelina
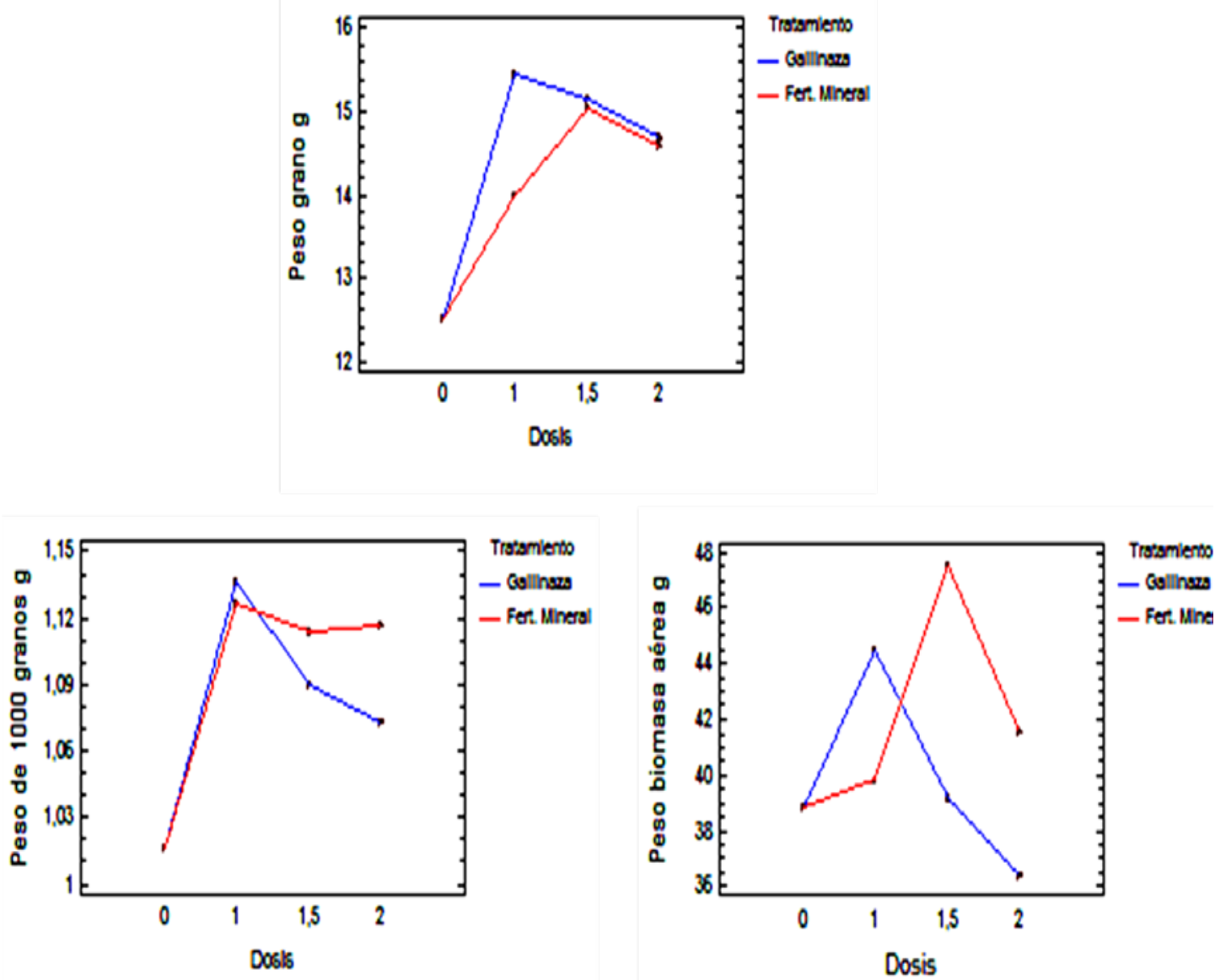
Figura 2. Interacción tratamiento (gallinaza o fertilizante mineral) * dosis $(0,1,1,5$ y 2), para porcentaje de nitrógeno Kjeldhal de: grano y biomasa aérea de camelina y para porcentaje de: proteína bruta y grasa bruta en grano de camelina
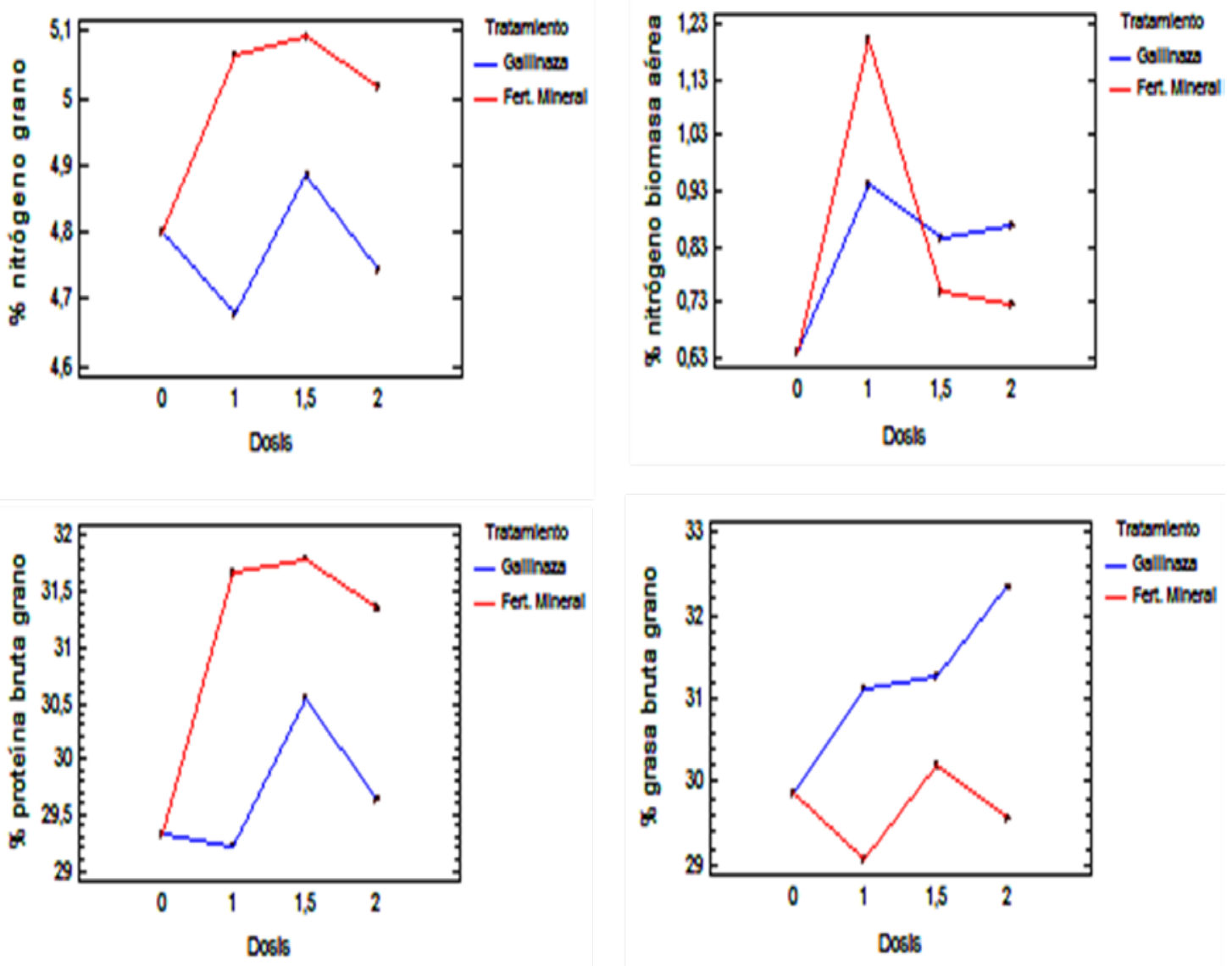

En el Cuadro 6 se presenta la comparación de dosis dentro de cada tratamiento para las variables que presentaron interacción. Las dosis fueron significativas $(P<0.05)$ en las variables peso biomasa aérea y \% $\mathrm{N}$ en biomasa aérea para gallinaza y para mineral también, alcanzándose los mayores valores para el caso de peso de biomasa área de $44.49 \mathrm{~g}$ en la dosis 1 para gallinaza y de $47.51 \mathrm{~g}$ en la dosis 1.5 para fertilizante mineral, mientras que para el caso del $\mathrm{N}$ en biomasa aérea los mayores valores se alcanzaron en la dosis 1, siendo de 0.94 y $1.21 \%$ para ambos tratamientos respectivamente. Estos resultados se ajustan a la Ley de los rendimientos menos que proporcionales, de los rendimientos menguantes o de Mitscherlich ${ }^{(22)}$ que dice: cuando se suministran dosis crecientes de abonos, los aumentos de cosecha obtenidos son cada vez menores a medida que las dosis aumentan. En el

caso del primer cultivo de cebada la dosis idónea fue $\mathrm{d} 1$, siendo las siguientes dosis superiores a la idónea, y después de dicho cultivo la cantidad de nutrientes residuales en el suelo (iniciales en el cultivo de camelina) continuó cumpliendo dicha ley.

La variable \%PBG fue solo significativa en el tratamiento de fertilización mineral, llegando a un valor máximo de $31.82 \%$ en la dosis 1.5 , mientras que para \%GBG sólo fue significativa para el tratamiento de gallinaza, alcanzando un valor máximo de $32.35 \%$ en la dosis 2, datos que concuerdan con los resultados obtenidos por otros autores $^{(10,14)}$, que observaron cómo al utilizar una fertilización mineral y al aumentar las dosis de fertilización, se produjo un aumento de la proteína en grano y una disminución del contenido en grasa bruta. Esta disminución en grasa bruta también se 
Cuadro 6. Comparación de dosis de gallinaza de gallina o fertilizante mineral para las diferentes variables

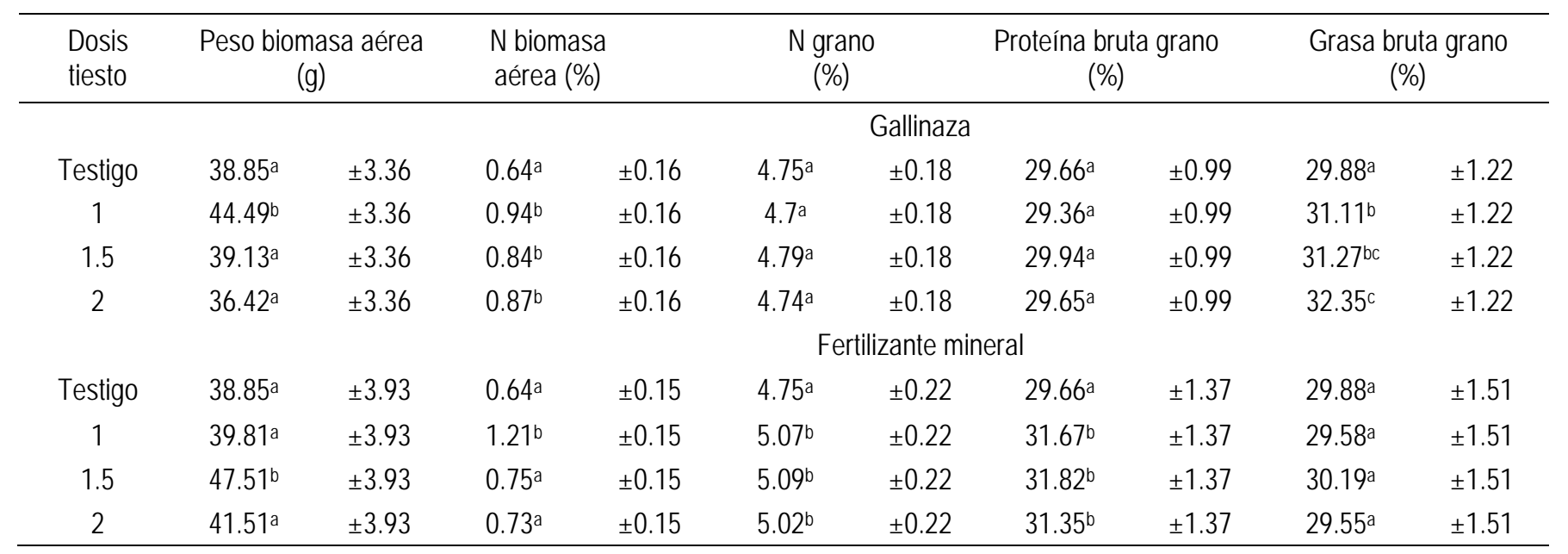

abc Valores de cada columna seguidos de letras diferentes indican diferencias significativas entre dosis $(P<0.05)$.

\pm Error estándar.

observó en el presente ensayo con la fertilización mineral en comparación con los valores obtenidos para gallinaza. Siendo los mayores valores para \%PBG con el tratamiento de gallinaza de $29.94 \%$ frente al $31.82 \%$ en el tratamiento de fertilización mineral, mientras que para el \%GBG, con el tratamiento de gallinaza fueron de 32.35 vs $30.19 \%$ en fertilización mineral $(P<0.05)$. Dichas diferencias se pueden observar en las gráficas de interacción de ambas variables (Figura 2).

Estos efectos también fueron observados por otros autores ${ }^{(8,11)}$, los cuales en ensayos de campo con camelina en localidades del centro sur de Chile para estudiar el efecto de la fertilización nitrogenada (0, 75, 150 y $300 \mathrm{~kg}$ nitrógeno/ha), observaron una correlación negativa entre el contenido en aceite y la fertilización nitrogenada. En el presente ensayo se observa un efecto positivo entre el contenido en aceite (expresado como grasa bruta) y la fertilización con gallinaza.

Según se muestra en el Cuadro 7 los mayores valores obtenidos en el peso 1,000 granos variaron entre $1.02 \mathrm{~g}$ para el testigo y 1.13 para la dosis 1 , valores próximos a los obtenidos en ensayos en tiestos para estudiar el efecto de la fertilización con nitrógeno con cv. Calena, observando efectos

Cuadro 7. Promedios de rendimientos agrícolas equivalentes en $\mathrm{kg} / \mathrm{ha}$, en grano

\begin{tabular}{llllllll}
\hline Tratamiento-Dosis & $\begin{array}{l}\text { Peso 1,000 } \\
\text { granos }(\mathrm{g})\end{array}$ & $\begin{array}{l}\text { Peso grano } \\
\text { (g/tiesto) }\end{array}$ & $\begin{array}{l}\text { Peso grano } \\
\text { (kg/ha) }\end{array}$ & PBG (\%) & PBG (kg/ha) & GBG (\%) & $\begin{array}{l}\text { GBG } \\
(\mathrm{kg} / \mathrm{ha})\end{array}$ \\
\hline GP- d0 & 1.02 & 12.52 & 1252 & 29.66 & 371.34 & 29.88 & 374.10 \\
GP- d1 & 1.1 & 15.47 & 1547 & 29.36 & 454.19 & 31.11 & 481.27 \\
GP- d1.5 & 1.06 & 15.20 & 1520 & 29.94 & 455.08 & 31.27 & 473.74 \\
GP- d2 & 1.07 & 14.68 & 1468 & 29.65 & 435.26 & 32.35 & 474.90 \\
\hline FM- d0 & 1.02 & 12.52 & 1252 & 29.66 & 371.34 & 29.88 & 374.10 \\
FM- d1 & 1.13 & 13.98 & 1398 & 31.67 & 442.75 & 29.58 & 413.52 \\
FM- d1.5 & 1.11 & 15.04 & 1504 & 31.83 & 478.72 & 30.19 & 454.06 \\
FM- d2 & 1.12 & 14.53 & 1453 & 31.35 & 455.52 & 29.55 & 429.36 \\
\hline
\end{tabular}

Proteína bruta (\% PBG) y grasa bruta (\% GBG), obtenidos en camelina con fertilización residual de: gallinaza de gallina ponedora (GP): dosis 0 (d0), dosis 1 (d1), dosis 1.5 (d1.5) y dosis 2 (d2) o de fertilizante mineral (FM): d0, d1, d1.5 y d2. 
positivos en el peso de 1,000 granos y obtuvieron pesos entre 1.04 y $1.17 \mathrm{~g}^{(23)}$. En ensayos en campo ${ }^{(3)}$ con esta misma variedad y sin fertilización, obtuvieron para peso de 1,000 granos valores de 1.378 y $1.373 \mathrm{~g}$ en las parcelas no regadas y 1.390 y $1.381 \mathrm{~g}$ en las regadas; estos pesos obtenidos en campo fueron superiores a los obtenidos en tiestos de este ensayo, al tener la planta condiciones más idóneas para su desarrollo; aunque otros autores ${ }^{(1)}$ en ensayos también de campo con camelina en localidades del centro sur de Chile con distintas fechas de siembra y tres cultivares (cv. Gold of Pleasure, cv. Suneson y cv. Blaine Creek), obtuvieron para peso de 1,000 semillas valores entre 1.08 y $1.49 \mathrm{~g}$.

En el caso de la variable peso grano se encontraron diferencias significativas entre el testigo (dosis 0) y el resto de las dosis, siendo la dosis 1 con gallinaza donde se obtuvieron los valores más elevados.

Se realizó un estudio sobre los promedios de rendimientos agrícolas equivalentes en $\mathrm{kg} / \mathrm{ha}$, para peso del grano, proteína bruta y grasa bruta (Cuadro 7). Estos rendimientos equivalentes en grano, proteína bruta y grasa bruta se calcularon teniendo en cuenta el peso de grano obtenido para la superficie del tiesto de $0.1 \mathrm{~m}^{2}$ y se extrapoló a una hectárea. Se puede observar que los mayores rendimientos en grano se obtuvieron para gallinaza en dosis 1 y para fertilizante mineral dosis 1.5 que fueron de 1,547 y $1,504 \mathrm{~kg} / \mathrm{ha}$ respectivamente, datos que igual que para la biomasa se ajustan a la Ley de Mitscherlich(22); en este parámetro la mejor dosis en fertilizante mineral después del primer cultivo fue para la dosis 1.5. Para proteína bruta se obtuvieron los mayores rendimientos con gallinaza en dosis 1.5 y fertilizante mineral en dosis 1.5 que fueron de 455.08 y $478.72 \mathrm{~kg} / \mathrm{ha}$ respectivamente. Para grasa bruta se obtuvieron los mayores rendimientos con gallinaza dosis 1 y fertilizante mineral dosis 1.5 que fueron de 481.27 y 454.06 $\mathrm{kg} / \mathrm{ha}$ respectivamente.

Con los rendimientos equivalentes descritos en este trabajo, se pueden comparar con los de otros autores ${ }^{(2)}$, aunque se obtuvieron resultados diferentes debido al lugar del ensayo, época de siembra, etc. En experiencias de cultivo en campo con camelina cv. Calena en el Bajo Aragón de España, en secano con siembra otoñal, obtuvieron rendimientos bajos en grano de $500 \mathrm{~kg} / \mathrm{ha}$ debido a la escasa precipitación; o en ensayos de campo ${ }^{(4)}$ en una localidad de Rumania con dos fechas de siembra y tres cultivares de camelina, cv. Camelia, cv. Lindo y cv. Calena, obtuvieron rendimiento en grano para cv. Calena en siembra otoñal de 1,083 kg/ha y en siembra primaveral de $800 \mathrm{~kg} / \mathrm{ha}$. Este ensayo de camelina se realizó en siembra otoñal y los rendimientos equivalentes fueron superiores a los obtenidos por estos autores, aunque en ensayos ${ }^{(9)}$ en parcelas en el este de Canadá con dos líneas de camelina CDI005 y CDI 1007 para evaluar el efecto de la fertilización con nitrógeno en distintas dosis y azufre combinado, el óptimo de dosis de $\mathrm{N}$ fue entre 120 (dosis equivalente a la dosis 2 del primer cultivo) y $160 \mathrm{~kg} / \mathrm{ha}$. Estos autores obtuvieron unos rendimientos medios en grano para CDI005 y CDI 1007 de 1,638 y 1,911 kg/ha y en rendimiento en aceite de 591 y $715 \mathrm{~kg} / \mathrm{ha}$ respectivamente, y otros $^{(6)}$ que aplicaron estiércoles de pollo de engorde en forma de pellets en ensayos de campo en Apelsvoll, Noruega en cultivo de camelina y distintos niveles de fertilización de nitrógeno 0, 40, 80 y 120 $\mathrm{kg}$ nitrógeno/ha, obtuvieron rendimientos medios de grano de 1,504, 1,858, 1,928, 1,996 kg/ha respecto a cada dosis de fertilizante. En el presente ensayo, se obtuvieron rendimientos máximos equivalentes de $1,547 \mathrm{~kg} / \mathrm{ha}$ con el residual de gallinaza en dosis 1 (cuando se aplicó al suelo la gallinaza en el primer cultivo, equivalía esta dosis 1 a 60 kg nitrógeno/ha), valor inferior a los obtenidos por esos autores ${ }^{(6)}$.

En cuanto a rendimientos del cultivo, los resultados son intermedios y concordantes con ensayos $^{(3)}$ que realizaron en campo cerca de Bucarest con camelina cv. Calena y siembra a finales de julio, como segundo cultivo después de triticale, sin aplicar fertilización mineral, donde obtuvieron rendimientos en las parcelas sin riego entre 494.2 y $800.5 \mathrm{~kg} / \mathrm{ha}$ y en las regadas entre $1,104.3$ y 1,203 $\mathrm{kg} / \mathrm{ha}$. En el presente ensayo los rendimientos para grano con residual de gallinaza fluctuaron entre $1,547 \mathrm{~kg} / \mathrm{ha}$ para d1 y $1,468 \mathrm{~kg}$ para d2 y con residual de mineral entre 1,398 kg para d1 y 1453 
$\mathrm{kg}$ para d2. Sin fertilización el rendimiento equivalente en grano fue de $1,252 \mathrm{~kg} / \mathrm{ha}$.

Se observó una disminución en el porciento de grasa bruta con la dosis 1 y 2 de fertilizante mineral respecto al testigo dosis 0 , pero también se obtuvo un mayor rendimiento en grano con la fertilización mineral en las tres dosis ensayadas respecto al testigo, resultados que también observaron en ensayos de campo en el norte de Wyoming, USA ${ }^{(7)}$ con camelina para determinar el efecto de la aplicación de nitrógeno en dosis de 0, 28, 56 y 112 $\mathrm{kg}$ nitrógeno/ha, en el rendimiento, proteína y contenido en aceite del grano, donde observaron un incremento en el rendimiento y proteína con la fertilización nitrogenada y un descenso en el contenido en aceite respecto al tratamiento sin fertilización, pero consideraron que esta disminución se compensaba con el incremento en el rendimiento en grano obtenido.

\section{CONCLUSIONES E IMPLICACIONES}

El cultivo de camelina cv. Calena en siembra otoñal obtuvo los mejores resultados con el tratamiento residual de gallinaza de gallina ponedora con la dosis 1 en cuanto a rendimiento en biomasa aérea y grano y en el peso de 1,000 granos. En cuanto a calidad del grano: para contenido en proteína bruta se obtuvo el mayor valor con mineral dosis 1.5 y respecto a contenido en aceite (grasa bruta) se obtuvo el mayor valor con gallinaza dosis 2. En general, la fertilización mineral residual sería la idónea en camelina si buscamos mayor porcentaje de proteína bruta y la residual de gallinaza si queremos mayor contenido en aceite (grasa bruta). La camelina podría ir en una rotación de cultivos como segundo cultivo, sin fertilizar, y aprovechar la fertilización orgánica (gallinaza de gallina ponedora) - fertilización mineral del cultivo anterior, abaratando costos de cultivo al no tener que fertilizar. La respuesta de la camelina a la fertilización residual orgánica y mineral fue en general positiva en las siete variables estudiadas. Para validar estos ensayos en tiestos e invernadero, se recomienda repetir estas experiencias en condiciones de campo.

\section{AGRADECIMIENTOS}

Este estudio se realizó gracias al proyecto RTA2011-00047-00-00 financiado por el INIAFEDER. Los autores agradecen a Jesús García su colaboración en las tareas de invernadero y recolección de datos.

\section{LITERATURA CITADA}

1. Berti M, Wilckens R, Fischer S, Solis A, Johson B. Seeding date influence on camelina seed yield, yield components and oil content in Chile. Ind Crop Prod 2011;(34):1358-1365.

2. Gutiérrez López $M$, Albalat Borrás A. El cultivo de la camelina en Aragón. Primeras experiencias de su cultivo en el Bajo Aragón.Tierras-Agricultura 2013; (208): 72-79.

3. Dobre $P$, Jurcoane $S$, Matei F., Stelica C, Farcas N, Moraru AC. Camelina sativa as a double crop using the minimal tillage system. Rom Biotech Lett 2014; 19(2): 9190-9195.

4. Toncea I. The seed yield potential of camelina- first Romanian cultivar of (Camelina sativa L. Crantz). Rom Agric Res 2014; (31):17-23.

5. Zubr J. Camelina oil in human nutrition. Agro Food Ind $\mathrm{Hi} \mathrm{Tec}$ 2009;20(4):22-28

6. Henriksen BIF, Lundon AR, Presttlokken E, Abrahamsen U, Eltun R. Nutrient supply for organic oilseed crops, and quality of potential organic protein feed for ruminants and poultry. Agron Res 2009; (7 Special issue II):592-598.

7. Sintim HY, Zheljazkov VD, Obour AK, Garcia y Garcia A, Foulke TK Influence of nitrogen and sulphur application on camelina performance under dryland conditions. Ind Crop Prod 2015; (70):253-259.

8. Malhi SS, J ohnson EN, Hall LM, May WE, Phelps S, Nybo B. Effect of nitrogen fertilizer application on seed yield, $\mathrm{N}$ uptake, and seed quality of Camelina sativa. Can J Soil Sci 2014;(94):35-47.

9. J iang Y, Caldwell CD, Falk KC. Camelina seed quality in response to applied nitrogen, genotype and environment. Can J Plant Sci 2014;94(5):971-980.

10. Urbaniak SD, Caldwell CD, Zheljazkov VD, Lada R, Luan L The effect of cultivar and applied nitrogen on the performance of Camelina satica L. in the Maritime Provinces of Canada. Can J Plant Sci 2008; 8(1):111-119.

11. Solis A, Vidal I, Paulino L, Johnson BL, Berti MT. Camelina seed yield response to nitrogen, sulphur, and phosphorus fertilizer in south Central Chile. Ind Crop Prod 2013; (44):132-138.

12. DOUE. Reglamento (UE) ํo 575/2011 de la Comisión de 16 de junio de 2011 relativo al Catálogo de materias primas para piensos. Diario Oficial de la Unión Europea. Luxemburgo. 2011;159:25-65.

13. Zubr J. Qualitative variation of Camelina sativa seed from different locations. Ind Crop Prod 2003; (17):161-169.

14. Johnson JMF, Gesch RW. Calendula and camelina response to nitrogen fertility. Ind Crop Prod 2013; (43):684-691. 
15. Miralles de Imperial R, Martín JV, Rodríguez C, Calvo R, Delgado MM. La aplicación de gallinazas en sorgo forrajero como cultivo energético. Rev Int Contam Ambie 2011;27(3):171-179.

16. Miralles de Imperial R, Martín JV, Calvo R, Delgado MM. Evaluación de nutrientes lixiviados bajo cultivo de maíz por aporte de granjas avícolas. ITEA 2007; 108(3):376-392.

17. Williams CM, Barker JC, Sims JT. Management and utilization of poultry wastes. Rev Environ Contam Toxicol 1999;(162):105-157.

18. Boletín Oficial del Estado. Real Decreto 506/2013, de 28 de junio, sobre productos fertilizantes. BOE 2013; (164):51119-51207.

19. Martinelli T, Galasso I. Phenological growth stages of Camelina sativa according to the extended $\mathrm{BBCH}$ scale. Ann Appl Biol 2011; (158):87-94.
20. Ministerio de Agricultura, Pesca y Alimentación. Métodos Oficiales de Análisis. Ministerio de Agricultura, Pesca y Alimentación, Madrid, España. Tomo III, 1994.

21. Ministerio de Agricultura, Pesca y Alimentación. Métodos Oficiales de Análisis. Ministerio de Agricultura, Pesca y Alimentación. Madrid. Tomo I. 1993.

22. Saña Vilaseca J, Moré Ramos JC, Cohí R. La gestión de la fertilidad de los suelos. Ministerio de Agricultura, Pesca y Alimentación. Madrid. 1996.

23. Lošák T, Hlusek J, Martinec J, Vollmann J, Peterka J, Filipcik R, et al. Effect of combined nitrogen and sulphur fertilization on yield and qualitative parameters of Camelina sativa (L.) Crtz. (false flax). Acta Agriculturae Scandinavica Section B, Soil Plant Sci 2011;(61):313-321. 